\title{
Les phases de l'évolution de l'Université Saint-Joseph à Beyrouth : les premières décennies (1875-1914)
}

\author{
Rafael Herzstein \\ Open University of Israel
}

RÉSUMÉ :

L’origine de l'Université Saint-Joseph de Beyrouth (USJ) remonte au Séminaire de Ghazīr fondé par les Pères jésuites en 1843. Le Collège de Ghazirr, destiné à former le clergé maronite local, est transféré à Beyrouth en 1875. Ce centre d'études supérieures prend le nom d'Université Saint-Joseph. Le Pape Léon XIII, dans son audience du 25 février 1881, confere le titre d'Université pontificale à l'USJ. L'article traite de l'histoire de l'USJ, premier grand établissement jésuite francophone de la région qui, à l'époque, porte le nom de "Syrie ». On évoque les différentes étapes de la création de l'Université Saint-Joseph de Beyrouth durant la période 1875 à 1914. L’objectif de cet article est de témoigner du rôle central que l'USJ a joué dans la formation des élites du pays pour la période étudiée. En effet, les anciens étudiants occupent les postes-clés et y assument des charges importantes notamment dans la fonction publique.

\section{ABSTRACT :}

The origins of Saint Joseph University in Beirut (SJU) go back to the seminary founded by the Jesuits in Ghazir in 1843. The Collège in Ghazìr, intended to train the local Maronite clergy, relocated to Beirut in 1875, where it took the name of Saint Joseph University. In an audience on February $25^{\text {th }}, 1881$, Pope Leo XIII gave the USJ the status of pontifical university. This article examines the history of the USJ, the first large francophone Jesuit institution in the region referred to at the time as "Syria." It will look at each of the different stages of the creation of Saint Joseph University in Beirut from 1875 to 1914. The author argues that the USJ played a central role in educating the elites of the region during that period. Students of the USJ went on to occupy important positions, particularly in the public service.

L'origine de l'Université Saint-Joseph de Beyrouth (USJ) remonte au Séminaire de Ghazīr fondé par les Pères jésuites en 1843. Le Collège de Ghazīr, destiné à former le clergé maronite local, est transféré à Beyrouth en 1875. Ce centre d'études supérieures prend le nom d'Université Saint-Joseph. Le Pape Léon XIII, dans son 
audience du 25 février 1881, confere le titre d'Université pontificale à l'USJ.

Notre article traite de l'histoire de l'USJ, premier grand établissement jésuite francophone de la région qui, à l'époque, porte le nom de "Syrie ". Nous évoquons les différentes étapes de la création de l'Université Saint-Joseph de Beyrouth durant la période 1875 à 1914. Quelle a été la contribution des anciens étudiants de l'USJ au développement du pays ? Que sont devenus les anciens étudiants, quelles fonctions et professions ont-ils occupées dans le pays ? L'objectif de cet article est de témoigner du rôle central que l'USJ a joué dans la formation des élites du pays pour la période étudiée. En effet, les anciens étudiants occupent les postes-clés et y assument des charges importantes notamment dans la fonction publique.

L'Université Saint-Joseph de Beyrouth, durant ses premières décades, est assez éloignée du modèle que nous connaissons de nos jours, mais elle n'en a pas moins rempli, dans son contexte spécifique, sa mission de propagatrice de savoir, de moteur culturel et communautaire. Elle a pu assumer avec succès son rôle de lien entre la rationalité humaine et les impératifs de la foi, facilitant ainsi l'insertion de générations d'étudiants maronites dans le monde moderne.

Le regroupement de toute la communauté maronite, sous la protection spirituelle de l'Église catholique romaine et de la France, avec comme résultante l'élaboration d'une stratégie de survie unique dans la région, basée presque unilatéralement sur l'aide et les soutiens extérieurs, transforme le Mont Liban durant la seconde moitié $\mathrm{du} \mathrm{XIX}^{\mathrm{e}}$ siècle en une sorte d'enclave catholique " étrangère " au monde musulman environnant. Dans ce processus, l'action de l'Université Saint-Joseph de Beyrouth tenue par les Jésuites est déterminante.

Aussi, les missionnaires jésuites possèdent presque tous la nationalité française et sont formés par l'Euvre des Écoles d'Orient. Fondée en 1865, cette institution a pour fonction d'assister les communautés religieuses catholiques établies au ProcheOrient, en diffusant, en particulier, l'enseignement du français et en participant à sa diffusion culturelle. En 1900, Jésuites et Lazaristes de la " Mission de Syrie " instruisent 12000 garçons, alors que des religieuses de diverses congrégations éduquent 5000 filles, en plus de l'activité de l'Université Saint-Joseph. ${ }^{1}$ Par leur intermédiaire se répand le français, qui s'impose comme la langue des études. La France, grâce à ses religieux, est alors la seule à offrir un enseignement "moderne ", c'est-à-dire en adéquation avec les besoins du temps.

\section{La fondation de l'Université}

Depuis 1820, une active mission protestante américaine est établie à Beyrouth. ${ }^{2}$ Elle y implante son imprimerie arabe, d'abord établie à Malte. Cette mission crée en 1866 le "Syrian Protestant College ", ${ }^{3}$ dans un parc de 10 hectares sur les hauteurs du Ras Beyrouth. Sa construction forme comme une cité à part. Il comporte un Collège, une École normale d'Instituteurs et un Séminaire de prédicants locaux, puis une École de médecine et de pharmacie. Le tout est largement subventionné par les protestants américains, jusqu'à ce que le gouvernement des États-Unis en fasse, en 1920, une Université. ${ }^{4}$ Cette institution confere au protestantisme une grande importance dans 
la région, et ce, grâce à la publication de nombreux ouvrages classiques et autres. En 1870, Beyrouth compte trois journaux arabes sur quatre. Ses principaux directeurs (Bliss, Van Dyck et Post), ${ }^{5}$ dont la valeur professionnelle et les talents d'organisateurs développés participent au rayonnement littéraire. L’outillage de leur imprimerie, longtemps la meilleure de l'Orient, y contribue également. ${ }^{6}$

Les Pères jésuites fondent en 1843 un Séminaire à Ghazīr destiné à former le clergé local. ${ }^{7}$ Des élèves laïcs sont accueillis dès 1849 dans la nouvelle section de français. En 1855, fut créé aussi un Collège et dès 1859 les élèves laïcs étaient plus nombreux que les Séminaristes. D'après les documents d'archives, le Collège de Ghazīr est transféré à Beyrouth en 1875..$^{8}$ Malgré sa prospérité, le "Collège Saint-Joseph " ne peut contrecarrer l'influence de la Mission protestante américaine; il faut pour cela une véritable université. Elle s’impose également pour ouvrir des débouchés à la jeunesse catholique.

C'est dans ce but que la Mission jésuite commence par transporter le Collège de Ghazīr à Beyrouth. Le Père Ambroise Monnot ${ }^{9}$ est envoyé comme supérieur de la Mission en 1869, mais l'argent manque. Le Pape autorise les Pères Monnot et François-Xavier Pailloux ${ }^{10}$ à entamer une tournée de quête auprès des catholiques en Angleterre et en Amérique, ils récoltent 300000 francs en six mois. Ce n'est pas assez, mais les Jésuites achètent déjà un terrain de $17000 \mathrm{~m}^{2}$, sur les premières pentes de l'Achrafieh. La transaction se fait par l'intermédiaire de M. Derwich Tyān, grand ami et procureur des Pères, au prix de 80000 francs qu'ils doivent emprunter et que le Père Monnot rembourse. ${ }^{11}$

La construction de l'Université commence en octobre 1874, sans permis officiel (Constantinople le refuse). Le grand édifice, de deux et trois étages, est terminé au bout de deux ans. Au Nord, face à la mer, il présente un long bâtiment à trois pavillons surélevés. L'aile médiane est formée par l'église. La façade est sobre, sans ornement, mais la saillie et les créneaux donnent une note élégante et discrète. ${ }^{12}$

Les élèves de Ghazīr déménagent en novembre 1875 pour gagner leurs nouveaux locaux scolaires. À la Noël 1875 l'église était déjà en fonction. ${ }^{13}$ Le Père Rémi Normand (successeur du Père Monnot) se trouvait à Rome dès janvier 1881. Ainsi il obtint du Pape Léon XIII ${ }^{14}$ la confirmation canonique du titre d'Université pour l'USJ, la faculté de conférer les titres académiques et le doctorat en philosophie et en théologie (audience du 25 février 1881). ${ }^{15}$ Il est décidé que pour la collation de ces grades, on se conformerait aux usages de l'Université Grégorienne à Rome. ${ }^{16}$

\section{Les œuvres d'enseignement}

L'USJ comprend l'enseignement primaire, l'enseignement secondaire et l'enseignement supérieur.

L'enseignement primaire se donne dans deux écoles : l'école de Dahdah, dans le groupe des bâtiments de l'Université, et celle du quartier de Romaile. D'autres écoles (dans les villages proches de Beyrouth et ailleurs dans le pays) sont à la charge de l'USJ

L'enseignement secondaire comprend l'enseignement secondaire classique, l'enseignement secondaire spécial, les leçons élémentaires et une école préparatoire. Dans 
la mesure du possible, l'enseignement est conforme aux programmes de France. A la fin de leurs études, les élèves peuvent se présenter devant un jury du gouvernement français et obtenir leur baccalauréat.

\section{Le Séminaire oriental érigé en Faculté de Philosophie et de Théologie(1881)}

Le Préposé général Roothaan (Supérieur de la Compagnie de Jésus) reçoit du Pape Grégoire XVI (1831-1846) la mission de créer un Séminaire oriental, afin de former une élite de prêtres et de missionnaires en Orient. C'est le 2 février 1846 que les RR. PP. Planchet et Riccadonna inaugurent le Séminaire Saint-François-Xavier à Ghazīr. ${ }^{17}$ Grâce à une allocation de la Propagation de la Foi, le Séminaire a été installé dans l'ancien Palais de l'Emir Hassan Chihāb (frère de l'Emir Bachīr). Vu l'augmentation du nombre d'élèves, il est agrandi en $1859 .{ }^{18}$

Il est ensuite transféré dans les nouveaux locaux à Beyrouth en octobre 1875. En 1877, Ghazīr devient un Noviciat qui accueille et forme les Frères débutants à leur fonction de missionnaire en Orient. ${ }^{19}$

À Beyrouth, les séminaristes constituent un groupe à part, avec leur propre directeur, leur règlement intérieur, des locaux et une chapelle à eux. Les élèves du Petit Séminaire (admission à 12 ans) suivent, quant à eux, le cours secondaire classique du Collège.

C'est le Pape Léon XIII qui, en 1881, érige le Séminaire en Université. L'ancien Séminaire prend dès lors le nom de Faculté de Philosophie et de Théologie. La collation des grades est inspirée du Collège romain. Le Pape veut ainsi développer en Orient les hautes études philosophiques et théologiques et étendre l'éducation ecclésiastique aux séminaires épiscopaux et aux scolasticats des Congrégations religieuses, considérés dès lors comme des annexes de l'Université. ${ }^{20}$

\section{L'enseignement supérieur}

\section{La Faculté française de médecine et de pharmacie (1883)}

La Faculté de Médecine est la création la plus originale, la plus importante et la plus féconde de l'USJ. D'après les Relations d'Orient, l'idée d'une Faculté de médecine apparaît bien avant 1883. Un missionnaire écrit en 1872: "Les Protestants américains viennent de fonder une école de Médecine; c'est une séduction pour les familles qui veulent avoir un fils médecin; c'est un grand péril religieux $"{ }^{21}$

Encore faut-il des professeurs, des ressources et l'appui du gouvernement français. Le consul de France, M. Patrimonio se laisse convaincre en 1876 par le Père Normand. Le Consul demande alors à l'attaché militaire de l'ambassade de parler à Gambetta. Parallèlement, le Père Normand se rend à Rome pour influencer le Pape Léon XIII. Celui-ci fait savoir au gouvernement français qu'il désire que le projet d'Université aboutisse. Les ministres français y sont favorables. Pourtant Gambetta et Jules Ferry ne sont pas des cléricaux ! Mais lorsqu'il s'agit de voter le budget des missionnaires, le gouvernement français estima, selon la formule de Gambetta, que "l'anticléricalisme n'est pas un article d'exportation ". ${ }^{22}$ Cette attitude s'inscrit dans la tradition de 1793 : les Jacobins veulent que le drapeau tricolore flotte sur les chapelles des aumôniers de consulats en Orient. 
D'après une lettre conservée dans les Archives de la Compagnie de Jésus à Rome, Barthélemy-Saint-Hilaire, ${ }^{23}$ ministre des Affaires étrangères, obtint du Parlement - avec l'agrément du chef de gouvernement, Jules Ferry — un crédit de 150000 francs sur le budget de 1882, pour la future Faculté. ${ }^{24}$ Il est écrit dans un article du journal le Figaro que 25000 francs y sont ajoutés pour le cabinet de physique par Gambetta (chef du cabinet et ministre des Affaires étrangères). ${ }^{25}$ Comme procureur de la Mission, le Père Mazoyer ${ }^{26}$ doit traiter avec plusieurs ministres des Affaires étrangères qui se succèdent rapidement : Freycinet, ${ }^{27}$ Duclerc et Jules Ferry. Pour la première année, l'État accorde 30000 francs pour le traitement des professeurs (ministre Duclerc). Le Père Normand reçoit le 15 septembre 1883, de Jules Ferry, la promesse que le gouvernement demanderait aux Chambres un crédit spécial et un projet de règlement sur l'admission des élèves et le diplôme. ${ }^{28}$ Deux mois plus tard, en 1883, après un accord entre le gouvernement et les Jésuites de "Syrie ", une École française de médecine ouvre ses portes dans les locaux dépendant de l'Université. ${ }^{29}$

Pour les études pratiques, les sœurs de Charité prêtent leur hôpital. Le rapporteur du budget, Spuller, ${ }^{30}$ propose l'année suivante un crédit de 80000 francs. Paul Deschanel ${ }^{31}$ soutint ce budget (combattu par Hanotaux) ${ }^{32}$ et la proposition fut votée. Malgré l'orientation laïque de la majorité, une allocation d'environ 100000 francs est ensuite accordée par les Chambres. ${ }^{33}$ Le titre de Faculté de médecine est octroyé en 1888 par le gouvernement Français. ${ }^{34}$

Cette nouvelle Faculté se voit agrandie d'une École de pharmacie en 1889.35 D'après le Bulletin annuel de la Faculté française de Médecine et de Pharmacie de Beyrouth, différents diplômes ont successivement été conférés par la Faculté de Beyrouth :

Un " Diplôme spécial français des colonies » est délivré de la session d'octobre 1887 à celle de novembre 1894 (inclusivement), au nom de la Faculté de Beyrouth. Ce diplôme créé spécialement pour la Faculté est signé par le ministre de l'Instruction publique de France. ${ }^{36}$ Les titulaires peuvent exercer dans les colonies mais pas sur le territoire français.

Des diplômes français signés du ministre et émanant de la Faculté de Lyon sont délivrés de la session de novembre 1894 à celle d'octobre 1897 (inclusivement). ${ }^{37}$ En effet, trois professeurs appartenant à la Faculté de Lyon siègent lors de ces sessions. Un Diplôme d'État français et un Diplôme d'État ottoman ${ }^{38}$ sont octroyés à la Faculté de Beyrouth de la session de 1898 (février 1899) à celle de novembre 1913 inclusivement, par un jury mixte franco-ottoman (notons que ce n'est qu'à partir du 21 juillet 1897 qu'est instaurée la distinction entre Diplôme d'État et Diplôme d'Université 39$)^{40}$

Le développement de la Faculté est rapide, avec une augmentation progressive des effectifs. ${ }^{41}$ Une maternité et une polyclinique obstétricale et gynécologique s'y ajoutent en 1896, puis un Institut antirabique en 1910. En 1896, il est question d'y adjoindre aussi une École de sages-femmes, mais son ouverture est retardée par le peu d'activité de la maternité. ${ }^{42}$

Le R.P. Cattin (Chancelier de la Faculté de médecine) prend en main le nouveau projet des Jésuites : l'ouverture d'un hôpital d'enseignement dirigé par les professeurs 
de la Faculté. ${ }^{43}$ L'idée est soutenue dans la presse française à partir de 1911 et des mécènes (Sociétés savantes, grandes compagnies) font des dons importants. ${ }^{44}$ Les travaux commencent au printemps 1914 sur un terrain de trois hectares mais ils sont interrompus par la guerre et le chantier est détruit. ${ }^{45}$

Seul un petit nombre d'étudiants peut fréquenter les premiers locaux de la Faculté, rue Huvelin. En 1896 apparaît la nécessité d'étendre les locaux. En 1897, on décide du transfert et on achète un terrain situe à l'entrée de la ville, sur la route de Damas. ${ }^{46}$ Mais, alors que la prise du pouvoir par les Jeunes-Turcs ${ }^{47}$ entraîne des restructurations internes importantes pour l'Empire ottoman, les autorités françaises s'inquiètent de l'ampleur des investissements. Malgré le refus du Parlement français de voter une augmentation des subventions accordées à la Faculté, les travaux sont cependant engagés en $1911 .^{48}$ Le 21 novembre 1911 a lieu la cérémonie de la pose de la première pierre. ${ }^{49}$ Le premier cours à la Rue de Damas se tient le 16 octobre 1912.50

\section{La Faculté orientale (1902)}

D'après les Lettres d'Or de l'USJ, des ecclésiastiques et des séculiers fréquentent l'USJ depuis 1900 pour se perfectionner sur place dans la connaissance des langues et des littératures orientales. ${ }^{51}$ Ils sont originaires d'Allemagne, d'Autriche-Hongrie, de Belgique, d'Espagne, de France, d'Italie, etc.

Le professeur Martin Hartmann développe en 1898 et en 1901 dans l'Orientalistische Literaturzeitung, des arguments scientifiques pour réclamer la fondation d'un Institut oriental pour l'étude de l'arabe dans une ville "syrienne " (Alep, Damas, Haman). Ce centre suit l'influence allemande, dans le contexte de l'entreprise du Bagdadbahn et de la volonté de l'Allemagne de s'implanter fortement en Orient. ${ }^{52}$ La Russie tsariste envisage l'éventualité d'une création analogue en "Syrie " vers la même époque. ${ }^{53}$

M. Clermont-Ganneau (membre de l'Institut) propose en 1899 la fondation à Beyrouth " d'un établissement français de haute culture destiné à assurer en Syrie notre prestige et à y établir notre suprématie intellectuelle ". ${ }^{54}$ Diverses sollicitations, notamment celles de Rome, l'attente des Orientalistes européens, poussent l'USJ à créer des cours supérieurs de langues et de littératures orientales accessibles au public européen. La nouvelle institution s'appela Faculté orientale: les noms d'École orientale ou d'Institut oriental ne sont pas retenus de façon à ne pas susciter de comparaison avec les prestigieux Institut français d'Archéologie orientale du Caire et École d'Athènes. ${ }^{55} \mathrm{La}$ Faculté orientale est fondée officiellement en $1902 .^{56}$

En attendant l'aménagement de locaux spécifiques à l'intérieur de l'Université, l'amphithéâtre de l'ancienne École de médecine accueillit les cours de la nouvelle Faculté.

En 1903, le professeur Georges Kampffmeyer salue l'ouverture de la Faculté orientale (la nouvelle "création des Pères de Beyrouth, qui ont si bien mérité des études orientales ${ }^{57}$ ) dans l'Orientalistische Literaturzeitung. La prépondérance accordée à l'arabe dans le programme devait, à son avis, 
être approuvée sans réserve. Ceux qui connaissent les services des Jésuites en ce domaine de l'orientalisme, savent comment ils unissent l'étude approfondie de la littérature arabe à linitiation la plus étendue au savoir occidental...Quelle meilleure occasion -et sous quelle direction plus qualifiée-d'apprendre sur place à connaître l'Orient? Pour toutes les questions scientifiques, nous pouvons être certains de rencontrer, chez les Jésuites, la compréhension la plus large et une aide efficace. ${ }^{58}$

C'est en juin 1906 qu'un jury composé de professeurs de la Faculté orientale entend les premières soutenances de thèses.

La Faculté inaugure aussi en 1906, les Mélanges de la Faculté orientale comme son organe de publicité. La Faculté orientale devient plus importante. Ses représentants sont invités à des réunions internationales. Ainsi en 1905 le Congrès archéologique d'Athènes invite le Père Louis Jalabert ; d'autres professeurs de la Faculté sont invités à Berlin au Congrès des Sciences Historiques de 1908 et, la même année, au Congrès des Orientalistes de Copenhague. Les Mélanges ont ensuite publié les communications des représentants de la Faculté orientale présentées à ces deux congrès. ${ }^{59}$

Les Mélanges constituent un recueil à peu près annuel de travaux érudits, depuis 1906 : mémoires originaux, puis, à partir de 1909, bibliographies (comptes rendus critiques). Dans les listes d'abréviations, on voit bientôt apparaittre le sigle $\mathrm{MFOB}^{60}$ en tête des ouvrages d'orientalisme. Il est remplacé en 1922 (tome VIII) par le sigle MUSJ ${ }^{61}$ Le Docteur Forget écrit dans Le Museon de Louvain : «la guerre qui a interrompu tant de travaux utiles aura aussi marqué la fin de la Faculté orientale; les Mélanges, si hautement et si justement appréciés, sappelleront désormais Mélanges de l'Université Saint-Joseph».62

On peut se faire une idée de la collection entière à partir du premier volume de 1906, dont les sujets sont aussi variés qu’importants. On peut y lire : les thèses de doctorat des PP. Power et Hartigan, présentées lors de la première soutenance de thèse en juin 1906;

1. un article du Père Lammens sur la «Syrie » et le «Liban »;

2. un article du Père Mallon sur l'Égypte au Moyen-âge;

3. des écrits archéologiques et d'épigraphie des PP Sébastien Ronzevalle et Jalabert; ${ }^{63}$

4. une étude de M. Chaine sur les apocryphes éthiopiens;

5. un mémoire du Père Cheikho sur l'histoire des Croisades.

\section{La Bibliothèque orientale (1875)}

D'après une lettre conservée dans les Archives Secrètes du Vatican, la Bibliothèque orientale est fondée en même temps que l'Université, en $1875 .{ }^{64}$ Elle reprend les fonds du Collège-Séminaire de Ghazīr. C'est le Père Alexandre Bourquenoud, ${ }^{65}$ un fribourgeois décédé en mission en 1868, qui rassemble le premier noyau d'ouvrages en explorant et en inventoriant les richesses archéologiques de la région. ${ }^{66}$

Il s'agit d'un fonds d'environ 5000 volumes comprenant une collection d'ouvrages 
d'Orientalisme, d'Écritures saintes et de controverses, ainsi qu'une petite Bibliothèque orientale d'archéologie, de numismatique et de voyages. Le conservateur était le Père Van Ham, ${ }^{67}$ un Jésuite hollandais, venu en Syrie pour des études particulières, qui ensuite décide d'y rester jusqu'en 1889, date de sa mort. ${ }^{68}$

Sous l'impulsion du Père Louis Cheikho (Directeur de la bibliothèque de 1880 à 1927), l'institution connaît un nouvel essor. En 1894, elle prend le nom de Bibliothèque orientale dont le fonds est orienté vers les disciplines de l'orientalisme. ${ }^{69}$ D'une façon constante, le Père Cheikho enrichit le fonds, surtout en manuscrits mais aussi en imprimés, de telle sorte que la bibliothèque s'impose à l'attention générale et est regardée comme un indispensable instrument de travail pour toutes les questions orientales et d'antiquité classique.

Le développement de la Bibliothèque est favorisé par deux événements :

- la création en 1898 d'Al-Machriq (l'Orient), Revue catholique orientale de langue arabe, fondée par le Père Louis Cheikho qui la dirige jusqu’à sa mort (1927) ;

- la création en 1906 de la revue de la Faculté orientale, Les Mélanges de la Faculté orientale. $^{70}$

Ces deux périodiques se sont maintenus et ont prospéré. Ils permettent une « reconnaissance élargie auprès de tous ceux qui sintéressent à l'Orient ", ${ }^{71}$ comme l'écrit le professeur Georges Kampffmeyer, orientaliste allemand de l'Université de Marbourg. Les échanges avec les orientalistes européens viennent en outre enrichir la bibliothèque.

Alors qu'elle n'était au départ qu'une annexe de la Bibliothèque générale de l'Université (elle n'en fut séparée qu'en 1905), il est écrit dans les Archives de la Compagnie de Jésus à Rome que la Bibliothèque orientale s'est largement agrandie (surtout depuis la création de la Faculté orientale), par les dons de l'Académie des Inscriptions et Belles-Lettres et du gouvernement français. ${ }^{72}$ On retient principalement le legs de la Bibliothèque d'Antonin Goguyere, orientaliste français mort à Mascate. ${ }^{73}$ En 1905, sous le rectorat du Père Henri Gressien, ${ }^{74}$ la Bibliothèque orientale, dans ses propres locaux, rédige son règlement. ${ }^{75}$ La Bibliothèque orientale dispose dès lors de l'espace précédemment occupé par la Bibliothèque générale (plus une chambre pour les manuscrits). ${ }^{76}$

La Bibliothèque devient la plus importante de l'ensemble du Proche-Orient et, lors du déclenchement de la guerre en 1914, suite à l'expulsion des Jésuites, elle est protégée par les Consuls d'Allemagne, d'Autriche-Hongrie et des États-Unis auprès du gouvernement ottoman. ${ }^{77}$

\section{L'École de droit (1913)}

C'est en 1910 que Paul Joubin, Recteur de l'Académie de Lyon, exprime au Conseil de l'Université l'intérêt que présente l'expansion en Orient de l'École de droit. ${ }^{78}$ Les responsables lyonnais hésitent sur le lieu de l'implantation. Paul Huvelin dirige une première mission en 1911, qui visite Athènes, Beyrouth, Constantinople et Smyrne. 
Une deuxième mission aura lieu en 1912. Du fait de l'existence de la Faculté française de médecine et des intérêts lyonnais dans la soie du Mont-Liban, c'est Beyrouth qui est choisie. ${ }^{79}$ Les Jésuites acceptent de diriger une École de droit et une École d'ingénieurs " dans les mêmes conditions que la Médecine ». ${ }^{80}$

Pourtant, la situation est différente. C'est directement avec une université française que les Jésuites doivent négocier cette fois-ci. L'entreprise est si difficile que le recteur Joubin évite de rencontrer les Pères Chanteur ${ }^{81}$ et Chauvin de passage à Lyon. Les Jésuites refusent de servir de " prête-nom " à l'Université de Lyon qui se réserve les directions essentielles de la future École. ${ }^{82}$ C'est la nomination des enseignants qui pose le plus de problèmes. Paul Huvelin finira par trouver la solution : la fondation conjointe de l'École par l'USJ et une Association ad hoc, l'Association lyonnaise pour le développement de l'Enseignement supérieur et technique à l'étranger. Celle-ci représente l'Université de Lyon et comprend aussi des membres de la Chambre de commerce de Lyon. Elle sert d'intermédiaire entre les deux partenaires.

Le premier bureau élu de l'Association comprendra :

1. le Recteur Paul Joubin, président (le Professeur Huvelin lui succède dès 1914);

2. M. Ennemond Morel (vice-président de la Chambre de Commerce de Lyon), premier vice-président;

3. M. Louis Josserand (professeur à la Faculté de droit), deuxième vice-président;

4. le Dr Guiart (professeur à la Faculté de médecine), secrétaire général;

5. M. Louis Guerin (de la Chambre de commerce de Lyon), trésorier; ${ }^{83}$

En l'absence d'un accord global à la veille de l'ouverture de l'École, les Jésuites se réservent encore le droit de se retirer définitivement :

" le gouvernement et l'Université de Lyon nous ont ignorés. À Paris et à Lyon, les orateurs officiels n'ont même pas osé risquer une allusion aux collaborateurs sans lesquels les Écoles seraient encore à l'état de projet et peut-être pour longtemps $»{ }^{84}$

En 1913, l'École de droit s'installe au premier étage du bâtiment dans les locaux de la Faculté de médecine, qui elle-même déménage pour d'autres plus vastes. ${ }^{85}$ La leçon inaugurale prononcée le 14 novembre 1913 par Paul Huvelin porte sur l'École de droit de Beyrouth, fleuron académique de "l'Empire romain ». ${ }^{86}$ L'avantage d'une implantation juridique à Beyrouth est renforcé par l'intérêt manifeste pour la tradition du droit romain et du droit comparé. Dans l'esprit des fondateurs de l'École, Beyrouth symbolise la vivacité du droit romain, qui n'est pas une législation morte car ses principes déterminaient encore toutes les législations modernes. ${ }^{87}$ L'étude de cette matière était une bonne méthode pour comprendre l'évolution juridique des sociétés. Par ailleurs, il est écrit dans Les Jésuites en Syrie que le droit comparé et le droit romain sont propices à la coopération internationale. La mission de la nouvelle École est de propager en Orient les traditions de Rome et de la France. ${ }^{88}$ L'École de droit est réquisitionnée le 14 novembre 1914. ${ }^{89}$ La session d'examen de novembre 1914 ne peut se tenir qu'en février $1919 .^{90}$ 


\section{L'École d'ingénieurs (1913)}

La création en 1913 d'une École française d'ingénieurs suit la même formule : Association lyonnaise pour le développement de l'enseignement supérieur et technique à l'étranger et la Compagnie de Jésus. ${ }^{91}$ Dès 1880, le Consul de France à Beyrouth en lance l'idée. Mais des problèmes particuliers attendent les fondateurs : l'École "n'avait pas derrière elle, dans le milieu local, des traditions aussi respectables que l'Ecole de Droit [...] : pas d'atmosphère scientifique que l'on puisse comparer à ces gôuts juridiques et littéraires, si faciles à réveiller dans le Proche-Orient ". ${ }^{92}$ L'École est alors fondée sous la forme provisoire d'une École préparatoire, avec 17 candidats admis. ${ }^{93}$ L'inauguration des deux Écoles de droit et d'ingénieurs se tient en novembre 1913, dans les anciens locaux de la Faculté de médecine. ${ }^{94}$ C'est la Chambre de commerce de Lyon qui permet la fondation dont le financement est assuré, pour moitié, par les recettes propres de l'École et pour l'autre moitié par des subventions de la Chambre de commerce de Lyon, du ministère des Affaires étrangères ${ }^{95}$ et de l'Université de Lyon. $^{96}$

Évoquons à présent deux œuvres dont le but premier n'est pas l'enseignement, mais qui sont créées lors de la fondation de l'Université Saint-Joseph : l'observatoire de Ksara et l'imprimerie catholique.

\section{L'observatoire de Ksara (1907)}

Tout au long de l'histoire les missionnaires jésuites ont pour tradition de créer des observatoires car l'autorité que conferent les recherches scientifiques contribue à renforcer celle de leur enseignement et de leur prédication. ${ }^{97}$

C'est durant l'année 1903-1904 qu'apparaît à l'USJ le projet d'observatoire en "Syrie ". Il est ensuite à l'étude pendant deux ans et, le 4 novembre 1906, le Père Berloty $^{98}$ (qui a déjà travaillé à des observations astronomiques à Tortosa) reçoit la mission de créer le nouvel observatoire. Il voyagea dans les différentes propriétés jésuites en "Syrie " avant de choisir la propriété viticole de Ksara. ${ }^{99}$

L'emplacement du site de Ksara présente de nombreux avantages : accès facile, altitude élevée (920 mètres), ciel pur, climat sain, isolement, vue sur le « Liban » et sur l'anti-Liban, Ksara est établi à une trentaine de kilomètres à vol d'oiseau de Beyrouth, sur l'un des derniers contreforts du "Liban » et domine la plaine de la Bekaa. La grande route de Damas en facilite l'accès. ${ }^{100}$

Le Père Berloty arrive le 29 octobre 1907 à Ksara et, avec les instruments achetés en France et en Angleterre, il se met aussitôt au travail et ainsi les premiers bâtiments sont rapidement installés. ${ }^{101}$

\section{L'imprimerie catholique (1852)}

La Mission protestante américaine tire de son imprimerie arabe, la plus belle que possédait alors la "Syrie ", un avantage certain. De même qu'ils ont fondé l'USJ pour contrer l'influence de l'Université américaine («The Syrian Protestant 
College " à l'époque), les Jésuites fondent une puissante imprimerie arabe face à l'imprimerie américaine. ${ }^{102}$

Il leur faut attendre jusqu'en $1847 .{ }^{103}$ Ils se contentent ensuite, jusqu'en 1852 , d'une petite autographie, qui sert à la publication d'une dizaine d'ouvrages par le Père Billotet. ${ }^{104}$ Un pèlerin de Terre Sainte répand l'Imitation de Jésus-Christ en Syrie et fit le don de 5000 francs en 1852 pour acheter une presse. L'imprimerie débute ainsi et publie 2000 exemplaires de l'Imitation en $1854 .{ }^{105}$

C'est le Frère Tallon ${ }^{106}$ qui dirige alors l'imprimerie, il y est secondé par un ouvrier libanais, As'ad El-Khoury, à qui le gouvernement français décerne le titre d'officier d'académie pour ses 50 ans de service (il meurt en 1902). L'imprimerie acquiert des caractères romains en 1855 , reçoit une deuxième et une troisième presse de l'CEuvre des Écoles d'Orient (en 1856 et en 1861), puis se procure une petite machine à vapeur de 3 chevaux en $1867 .^{107}$

Elle publie plusieurs livres d'enseignement religieux et de prières, puis en 1857 la grammaire arabe-française du Père Abougit, le vocabulaire français-arabe du Père Heury $^{108}$ (in 8,715 pp.), et le dictionnaire arabe-français du Père Cuche ${ }^{109}$ (cité très favorablement par les arabisants pour sa qualité et son originalité). Au cours de l'année 1860, l'imprimerie édite 30000 exemplaires (60 000 en 1863) parmi lesquels 50 ouvrages inédits. ${ }^{110}$

De 1869 à 1901, le directeur de l'imprimerie est le Frère Elias. ${ }^{111}$ Originaire de Caifa, ce musulman converti au catholicisme et formé à l'imprimerie de Paris fait de celle de Beyrouth la plus importante de l'Empire ottoman. Il y forme des ouvriers dès 1861 et prend de nombreuses initiatives, notamment une nouvelle fonte de caractères. ${ }^{112}$ Sa Bible arabe d'octobre 1875 lui vaut une médaille d'or à l'Exposition de 1878. "L'impression de l'ouvrage, prononça le jury, la perfection de ses caractères le mettait à part parmi toutes les publications arabes connues à cette époque ". ${ }^{113}$

Au départ, l'imprimerie ne dispose pas encore de locaux autonomes et occupe deux ou trois petites pièces dans la Résidence. ${ }^{114}$ Ensuite, en 1860, elle déménage dans un nouveau bâtiment. Comme les installations croissent, l'imprimerie s'installe en 1875 définitivement dans les nouveaux locaux de l'USJ. ${ }^{115}$

Les publications en arabe se divisent en trois groupes :

1. Les publications religieuses (200 volumes) : des Concordances, des Catéchismes, l'Imitation et la Bible, des livres d'ascétisme, de controverse, de dogme, de liturgie, de morale, de poésie, etc. ${ }^{116}$

2. Les publications classiques et philologiques (environ 200 volumes également) : des grammaires, des littératures, des manuels de géographie et d'histoire, des spécimens de calligraphie arabe pour la lecture des manuscrits, des vocabulaires, en 1883 le vocabulaire arabe-français du Père Belot ${ }^{117}$ (considéré encore aujourd'hui comme un classique). En 1885, les professeurs de Beyrouth entament la publication des vieux lexicographes et philologiques arabes. En 1899, l'imprimerie publie la grammaire arabe en deux volumes du Père Vernier, ${ }^{118}$ l'Arabic-English Dictionary du Père Hava, ${ }^{119}$ et surtout le dictionnaire en trois volumes à trois colonnes, entièrement rédigé en arabe, 
du professeur de rhétorique arabe à l'USJ Sa īìd Chartouni. Puis parait une suite d'anthologies et de chrestomathies pour étudier la langue arabe. Elles sont rapidement adoptées par les écoles d'Égypte, de Palestine, de Syrie. ${ }^{120}$

3. Les Publications périodiques : Al-Bachir, Al-Machriq et les Mélanges de la Faculté orientale de l'Université Saint-Joseph. ${ }^{121}$

Al-Bachir (le Messager ou le Nouvelliste) est un tri-hebdomadaire religieux, défenseur de la cause religieuse en Orient. ${ }^{122}$ Le Père Monnot ${ }^{123}$ crée en janvier 1870 Le Concile $d u$ Vatican, pour défendre le Concile contre les attaques violentes des trois journaux protestants de Beyrouth, ${ }^{124}$ sous la forme d'une petite brochure de 8 pages hebdomadaires en arabe. En 1871, le périodique reçoit le nom de Al-Bachir, prend le format d'un journal et double le nombre de ses pages. ${ }^{125}$

En 1888, le journal reçoit un diplôme d'honneur et une médaille d'argent du Comité romain du jubilé de Léon XIII. Le 1er janvier 1894, Al-Bachir adopte le format des grands journaux d'Europe. Lors de ses noces d'argent, en 1895, patriarches et évêques s'accordent à louer " son zèle, ses luttes pour la défense des intérêts chrétiens ainsi que la sûreté, le sérieux de ses informations ". ${ }^{126}$ Nous avons trouvé dans les Archives de la Compagnie de Jésus à Rome que le journal est parfois soumis à la censure de Constantinople. ${ }^{127}$

Al-Machriq (l'Orient) est une revue de recherches scientifiques, spécialisée dans le domaine des études orientales et publiée sous la direction des Pères de l'Université Saint-Joseph. ${ }^{128}$ Le premier numéro parait enfin le $1^{\text {er }}$ janvier $1898 .{ }^{129}$ La publication est mensuelle, en arabe (80 pages). ${ }^{130}$ On y trouve des articles importants et de qualité, sur des sujets historiques, littéraires, philosophiques, polémiques, religieux, scientifiques et une abondante bibliographie d'ouvrages publiés en Orient. ${ }^{131}$

Al-Machriq lance d'emblée un appel aux Orientaux : "Étudions notre histoire ". ${ }^{132}$ La revue attire ainsi l'attention du monde scientifique. ${ }^{133} \mathrm{M}$. Charles Nallino, professeur à l'Université royale de Rome, affirme en 1900 qu'il considère le Machriq comme " un des centres de culture les plus actifs de l'Orient arabe ». ${ }^{134}$

Précédemment, nous avons mentionné la revue les Mélanges de la Faculté orientale. Celle-ci publie un condensé des travaux les plus importants des élèves et des professeurs de cette Faculté.

Pendant la guerre, les fonds des magasins et les machines de l'Imprimerie Catholique sont dispersés dans deux imprimeries à Beyrouth et deux autres à Damas. ${ }^{135}$ La rupture diplomatique entre la France et l'Empire ottoman intervient le 2 novembre 1914. Le 4 novembre les enseignants laïcs étrangers et leurs familles quittent la région. Les bâtiments de l'USJ sont réquisitionnés le 14 novembre courant. ${ }^{136}$ Les religieux ressortissants d'États en guerre sont expulsés de l'Empire Ottoman le $21 .^{137}$

\section{Conclusion}

Durant les trente premières années de son fonctionnement, l'USJ accueille en moyenne de 450 à 550 élèves par an. ${ }^{138}$ Après la guerre de $1914-1918$, leur nombre 
passe à 600-800 élèves. ${ }^{139}$ Sont ainsi issus du Séminaire oriental trois patriarches (dont un pour les Maronites), une trentaine d'évêques et plus de deux cents autres prêtres. ${ }^{140}$ À noter, que six d'entre sont victimes du génocide des Arméniens. Cela signifie que selon les chiffres de $1931,{ }^{141}$ des prêtres instruits formés et influencés par la France exercent dans 200 villages.

Plus de 850 médecins reçoivent leur diplôme de Docteur à la Faculté de médecine et ce, depuis sa création jusqu'aux années $1930 .{ }^{142}$

\section{Dispersés dans tout l'O rient, de la Mer Noire au Golfe Persique, de la Méditerranée à la Perse, dans les îles de l'Archipel, en Egypte, au Soudan, au Maroc, dans les colonies africaines, en Extrême-Orient, et jusqu'en Amérique, partout où ils s'en vont sinstaller, ils portent avec l'exemple, le bon renom de l'USJ. ${ }^{143}$}

De nombreux spécialistes sont également formés par l'École de droit et l'École d'ingénieurs. ${ }^{144}$

Les chiffres ${ }^{145}$ sont parlants mais ne rendent pas assez compte de la profondeur de l'influence de l'USJ. En effet, on se rappelle qu'au départ l'Université est créée dans le but de répondre aux besoins de la communauté maronite, leur permettant l'accès à des fonctions dont ils sont exclus jusque-là, ${ }^{146}$ manière pour les Jésuites de contribuer à l'œuvre " civilisatrice " de la colonisation française. Mais à la fin de la Première Guerre mondiale, les aspirations nationales sont apparues. La Faculté orientale de l'USJ dont les enseignants les plus célèbres étaient les professeurs Lammens, Cheikho et Ronzevalle a joué un rôle prépondérant dans le développement de deux mouvements nationaux séparés : le syrien et le libanais ${ }^{147}$ et a ainsi exprimé l'idée en faveur de la création du "Grand Liban " en 1920. ${ }^{148}$ C'est alors que les classes moyennes cultivées sont appelées à prendre la tête de la nation nouvelle. ${ }^{149} \mathrm{Ce}$ n'est donc pas un hasard si le Liban est gouverné à différentes époques par un ancien étudiant de l'Université, de même qu'en sont issus aussi presque tous les présidents du Conseil, la majorité des ministres et des hauts fonctionnaires. Les anciens étudiants de l'USJ prennent ainsi une place importante parmi ceux qui secondent les dirigeants du mandat français de cette période. Ainsi, une continuité visible s'installe dans le devenir des étudiants de l'USJ.

\section{Notes}

1 Denise Bouche, Histoire de la Colonisation française. Tome 2 : Flux et reflux (18151962). (Paris : Fayard, 1991), 272.

2 Munir Antonios Bashshur, The Role of the Two Western Universities in the National Life of Lebanon and the Middle East : A Comparative Study of the American University and the University of Saint-Joseph, thèse de doctorat (education), University of Chicago, 1964, 43.

3 "Vers la même année 1866, un riche américain, M. Doggs, leur fit un don de 500000 francs, pour élever à l'extrémité de la presqu'île de Beyrouth le vaste ensemble de bâtiments scolaires connu sous le nom d'Université américaine [à cette époque, The Syrian Protestant 
College ]. Il comprend un Collège secondaire, une École de médecine et une École de théologie pour former des ministres et des prédicants indigènes ». (Révérend Père Michel Jullien. La Nouvelle Mission de la Compagnie de Jésus en Syrie (1831-1895). Tome II. (Tours : Imprimerie A. Mame et Fils, 1898), 259.

4 Munir Antonios Bashshur. op. cit., 44.

5 Pour plus de détails sur Bliss, Van Dyck et Post voir : Abd Al-Latif Tibawi, American Interests in Syria 1800-1901. (Oxford, 1966).

6 Pour plus de détails sur l'activité des missionnaires protestants dans la région voir entres autres : Adnan Abu-Ghazleh, American Missions in Syria. A Study of American Missionary Contribution to Arab Nationalism in $19^{\text {th }}$ Century Syria. (Vermont : 1990); Abd Al-Latif Tibawi, op. cit.; Fruma Zachs, The making of Syrian identity : intellectuals and merchants in nineteenth century Beirut. (Leiden : 2005); Fruma Zachs, "From the mission to the missionary: the Bliss family and the Syrian Protestant College (1866-1920)", Welt des Islams 45, 2 (2005) : 255-291; Fruma Zachs, "Toward a proto-nationalist concept of Syria? Revisiting the American Presbyterian missionaries in the nineteenth-century Levant", Welt des Islams 41, 2 (2001) : 145-173.

7 Pour plus de renseignements sur la formation du clergé autochtone au Séminaire de Ghazìr voir : A.J.V., Carton RPO 85: Ghazīr, Différentes lettres.

8 A.R.S.I., Carton 1005 : 1869-1890, Mission Syria, Farde Syria 1005-I, Lettre numéro 35 du Père Monnot datant de 1875, Ibid., Farde Syria 1005-VI, Différentes lettres sur le passage de Ghazīr à Beyrouth, Ibid., Farde Syria 1005-XII, Différentes lettres sur le passage de Ghazīr à Beyrouth ;

A.P.F., Carton numéro 2 : 1874-1880 : Scritture Riferite nei Congressi Siria, Lettre : "Aperçu du R.P. Monnot, Supérieur Général de la Mission";

A.J.V., Carton RPO 21 : Histoire et présentation de la Mission de Syrie, Chemise 1 : Documents anciens, Numéro 11 : «Missions des Jésuites en Syrie, vue d'ensemble des œuvres entre 1875 et 1880 ".

9 Père Ambroise Monnot : né en 1831, jésuite en 1846, décédé en 1898 à Lyon. De 1869 à 1876, supérieur de la Mission de "Syrie ». Fondateur de l'USJ; à l'origine du Bachir, de la Bible arabe. Provincial de Lyon de 1877 à 1883; procureur de la Province et de la Mission de 1883 à 1896. (Henry Jalabert, sj, Jésuites au Proche-Orient. Notices biographiques (Université Saint-Joseph - Faculté des Lettres et Sciences Humaines : Collection Hommes et Sociétés du Proche-Orient, 1986).

10 Père François-Xavier Pailloux : né en 1814, jésuite en 1838, décédé en 1887 à Lyon. De 1871 à 1877 : Compagnon du père Ambroise Monnot dans ses quêtes d'Amérique (1871-1873). Architecte de l'USJ. Operarius, écrivain, architecte. (Henry Jalabert. sj. Ibid.).

11 ACG Beyrouth, Carton: A107, 1873/1874, Différentes lettres.

12 A.J.V., Collection PRAT, Tome 26 : Mission de Syrie 1875-1905, Université.

13 A.R.S.I., Carton 1005 : 1869-1890, Mission Syria, Farde Syria 1005-I, Différentes lettres du Père Ambroise Monnot.

14 Pape Léon XIII : Pape de 1878 à 1903. (Le Petit Larousse illustré, 1996).

15 A.J.V., Carton RPO 21 : Histoire et présentation de la Mission de Syrie, Chemise 2 : avant 1914, Lettre : "La Compagnie de Jésus en Orient : Prospectus »; A.R.S.I., Carton 1005 : 1869-1890, Mission Syria, Farde Syria 1005-II, Lettre numéro 26 datant de février 1881.

16 Pour plus de détails sur les raisons de la fondation de l'USJ voir : Rafael Herzstein, Université Saint-Joseph de Beyrouth, Fondation et fonctionnement de 1875 à 1914.

Bruxelles:2008; Rafael Herzstein, «Une réconciliation entre Paris et la Compagnie de Jésus au Levant (1875-1914) Faux-fuyant ou nécessité ponctuelle? ». Mémoire Spiritaine : Histoire, Mission, Spiritualité 22 (2005) : 99-113; Rafael Herzstein, " La France et le retour des jésuites au Levant au sein de la communauté maronite-Un 
nouveau modèle de compromis pédagogique ", Middle East Review of International Affairs (MERIA) Journal 2, 1 (2007) : 95-105; Rafael Herzstein, "The foundation of the Saint-Joseph University of Beirut : the teaching of the Maronites by the second Jesuit mission in the Levant ", Middle Eastern Studies 43, 5 (2007): 749-759; Rafael Herzstein, "Saint-Joseph University of Beirut : An Enclave of the FrenchSpeaking Communities in the Levant, 1875-1914 ", Itinerario 2, Vol. XXXII (2008) (International Journal on the History of European Expansion and Global Interaction) : 67-82; Rafael Herzstein, "Les pères jésuites et les Maronites du Mont Liban : l'Université Saint-Joseph de Beyrouth » Histoire \& Missions Chrétiennes 9, (mars 2009) : 149-175.

17 A.J.V., Carton RPO 49 : Séminaire Saint-François-Xavier à Ghazīr.

18 A.R.S.I., Carton 1005 : 1869-1890, Mission Syria, Farde Syria 1005-XVIII (Titre de ventes, d'achats), Lettre numéro 6.

19 Pour plus de renseignements sur le Collège de Ghazīr voir : A.J.V., Carton RPO 14 : Ghazīr, A.J.V., Carton RPO 85 : Ghazīr.

20 A.J.V., Carton RPO 21 : Histoire et Présentation de la Mission de Syrie, Chemise 2 : avant 1914, Lettre : "La Compagnie de Jésus en Orient : Prospectus".

21 "Syrie : La Faculté de médecine et son jubilé ». Relations d'Orient et suppléments, Tome I (bis) de 1908, 186.

22 Formule reprise par M. le député Coulondre le 26 novembre 1904 à la Chambre dans J.O. du 27 novembre 1904, compte-rendu de la séance du 26 novembre 1904, 2710.

23 Barthélemy-Saint-Hilaire, Jules, Homme politique et érudit français (Paris 1805-Paris 1895). Traducteur des œuvres d'Aristote. (Le Petit Larousse illustré, 1996).

24 A.R.S.I., Carton 1005 : 1869-1890, Mission Syria, Farde Syria 1005-VI, Lettre numéro 26 de Barthélemy-Saint-Hilaire datant du 2/6/1881.

25 Le Comte de Lubersac. "Une CEuvre française en péril », Le Figaro, mardi 26 décembre 1911.

26 Père Mazoyer : né en 1840, jésuite en 1861, décédé en 1918 à Cannes. De 1879 à 1880, Beyrouth : Préfet Spirituel. Une seule année de résidence au Proche-Orient, mais procureur de la mission à Paris (1880-1889) et à Lyon (1904-1913). D’un dévouement sans limites, presque aveugle, il a couru l'Europe pour ses quêtes. (Henry Jalabert, sj. op. cit.).

27 Freycinet, Charles, Louis de Saulses de. (Foix 1828-Paris 1932). Ingénieur et homme politique français. Plusieurs fois Président du Conseil et ministre des Affaires étrangères. (Le Petit Larousse illustré, 1996).

28 Voir à ce sujet : ACG Beyrouth, Carton: A137, 1885/86, Dossier: "Traitements des Professeurs ». Ibid., Carton: A235, 1913, Carton: A238, 1913, Carton: A293, 1903/04, Carton: A303, 1908/1909, Carton: A337, 1882/1914.

29 A.J.V., Collection PRAT, Tome 27 : Mission de Syrie, Maisons, F.F.M..

30 Spuller, Eugène : Homme politique français (1835-1896). Avocat (1862), ami de Gambetta. Député (1876-1892). Ministre de l'Instruction publique (1887), puis ministre des Affaires étrangères (1889-1890). Il entre au Sénat en 1892. Il fait preuve de souplesse avec l'Eglise, préconisant dans les rapports de la République avec celle-ci, un " esprit nouveau "; cette modération sera taxée par l'extrême gauche de cléricalisme. (Le Petit Larousse illustré, 1996).

31 Deschanel, Paul : Homme politique français (Schaerbeek 1855-Paris 1922). Président de la République (1920). (Le Petit Larousse illustré, 1996).

32 Hanotaux, Gabriel : Historien et homme politique français. (Aisne 1853-Paris 1944). Ministre des Affaires étrangères (1894-1898).

33 A.J.V., Collection PRAT, Tome 26 : Mission de Syrie 1875-1905, F.F.M. P. 254.

34 A.J.V., Collection PRAT, Tome 27 : Mission de Syrie, Maisons, F.F.M. 1888-1889.

35 A.J.V., Collection PRAT, Tome 26 : Mission de Syrie 1875-1905, F.F.M.. 
36 A.E., Turquie, Tome 27, Ecole de médecine de Beyrouth, 1888.

37 A.J.V., Collection PRAT, Tome 27 : Mission de Syrie, Maisons, F.F.M..

38 A.J.V., Collection PRAT, Tome 27 : Mission de Syrie, Maisons, F.F.M. Diplôme francoottoman 1897-1898;

ACG Beyrouth, Carton: A357, 1902-1914, Dossier: Note sur les Diplômes délivrés par la F.F.M et Pharmacie de Beyrouth.

39 ACG Beyrouth, Carton: A172, 1899, Dossier: Question de diplômes, Ibid., Carton: A357, 1902-1914, Dossier: Note sur les Diplômes délivrés par la F.F.M. et pharmacie de Beyrouth.

40 Note sur les Diplômes délivrés par la Faculté française de médecine et de pharmacie de Beyrouth. Bulletin annuel de la Faculté française de Médecine et de Pharmacie de Beyrouth. Années scolaires 1947-1948 et 1948-1949 (62 ${ }^{\text {ème }}$ et 63 ${ }^{\text {ème }}$ années), 21.

41 A.J.V., Collection PRAT, Tome 27 : Mission de Syrie, Maisons, F.F.M..

42 ACG Beyrouth, Carton: A342, 1899-1914, Dossier: F.F.M..

43 Le Comte de Lubersac. "Une CEuvre française en péril ». Le Figaro, mardi 26 décembre 1911.

44 Le Figaro. Ibid.

45 Pour plus de renseignements sur le projet d'ouverture d'un hôpital d'enseignement dirigé par les professeurs de la Faculté voir : ACG Beyrouth, Carton: A235, 1913, Dossier 3/2: Allocations, Ibid., Carton: A337, 1882-1914, Dossier: Subventions à l'hôpital;

A.S.V., Carton numéro 199: 1900-1913 : Relazioni e Corrispondenza della Delegazione Apostolica con i Padri Gesuiti, Lettre de 1911.

46 A.R.S.I., Registra Lugduni VIII, p. 99, Lettre du R.P. Martin du 24 avril 1898.

47 Les Jeunes-Turcs : Groupe d'intellectuels et d'officiers ottomans libéraux et réformateurs, d'abord rassemblés en diverses sociétés secrètes. Ils contraignirent le Sultan Abdülhamid II à restaurer la Constitution (1908) puis à abdiquer (1909) et dominèrent la vie politique ottomane jusqu'en 1918. (Le Petit Larousse illustré, 1996).

48 Ambassade Constantinople, Carton: E706, 1886/1890, Dossier: F.F.M. : construction de la nouvelle Faculté, 1902/1912.

49 Faculté de médecine de Beyrouth. Pose de la première pierre. Beyrouth, 1911.

50 ACG Beyrouth, Carton: A238, 1913, Dossier numéro 3: F.F.M., nouvelle Faculté; A.S.V., Carton numéro 199: 1900-1913 : Relazioni e Corrispondenza della Delegazione Apostolico con i Padri Gesuiti, Lettre d'invitation du Père Cattin datant de 1912.

51 Université Saint-Joseph de Beyrouth. Lettres d'Or. Tome II, 1908-1909. (Bruxelles : Editeur Joseph Polleunis, 1909), 67-68.

52 Pour plus de renseignements sur les intérêts allemands en Orient voir : Germany and the Middle-East, 1835-1939 : International Symposium, April 1975. Tel-Aviv : Tel-Aviv University, Faculty of Humanities, the Aranne School of History, Institute of German History, 1975.

53 Pour plus de renseignements sur les intérêts russes en Orient voir : Victor Morpurgo. Politique de la Russie en Orient, avenir de la Turquie : Documents. (Paris : D. Giraud, 1854).

54 Les Jésuites en Syrie, 1831-1931. Université Saint-Joseph. (Paris : Dillen, 1931), 12 fascicules. Fascicule 9: L'ouvre scientifque. Orientalisme. Archéologie, 21-23.

55 "La Faculté orientale des Jésuites en Syrie », La Croix, 8 juillet 1906.

56 A.J.V., Carton RPO 52 : Université Saint-Joseph, Faculté orientale.

57 Les Jésuites en Syrie, 1831-1931, op. cit., 24.

58 Ibid.

59 Ibid., 25.

60 MFOB initiales des Mélanges de la Faculté orientale de Beyrouth. 
61 MUSJ initiales des Mélanges de l'Université Saint-Joseph.

62 Le Museon de Louvain. Tome XXXVI de 1923. P. 318.

63 Père Louis (Al) Jalabert, né en 1877, jésuite en 1895, décédé en 1943 à Nice. Un peu plus de huit années à Beyrouth, en trois séjours : six ans, deux ans, trois mois. 1901-1907 : scolastique, professeur de $3^{\text {ème }}$ (1901-1902), puis étudiant l'épigraphie et l'enseignant à la Faculté orientale à partir de 1903. 1911-1913 : écrivain, de 1913 à 1914 «Troisième an » en Europe. À partir de 1914, professeur de rhétorique, écrivain. (Henry Jalabert, sj. op.cit.).

64 A.S.V., Carton numéro 198: 1830-1899 : Relazioni e Corrispondenza della Delegazione Apostolica con i Padri Gesuiti, Lettre de 1876.

65 Père Alexandre Bourquenoud : (Province de Haute-Allemagne, 1824-1868). Professeur de théologie, orientaliste. Né en Suisse romande. Jésuite en 1842. Envoyé en Orient étudier l'arabe et la géographie biblique. Préfet des études à Ghazīr en même temps que professeur de théologie. Il mourut en 1868 à Ghazīr. (Henry Jalabert, sj. op. cit.).

66 A.S.V., Carton numéro 198: 1830-1899 : Relazioni e Corrispondenza della Delegazione Apostolica con i Padri Gesuiti, Lettre de 1876.

67 Père Joseph Van Ham : (Province des Pays-Bas, 1813-1889). Ecrivain, polémiste. Néerlandais, né en Allemagne. Jésuite en 1835. Il arrive à Beyrouth en 1865 pour trois années d'étude des langues orientales, et obtient à la fin de cette période de rester dans la Mission de Syrie afin d'y collaborer à la version arabe de la Bible, pour laquelle il fournit un travail important. Il mourut en 1889 à Tanail. (Henry Jalabert, sj. op.cit.).

68 A.S.V., Carton numéro 198: 1830-1899 : Relazioni e Corrispondenza della Delegazione Apostolica con i Padri Gesuiti.

69 A.S.V., Ibid.

70 Les Jésuites en Syrie, 1831-1931, op. cit., 9-10.

71 Ibid.

72 A.R.S.I., Carton 1007 : 1901-1918, Mission Syria, Farde Syria 1007-I, Différentes lettres du Père Cattin.

73 A.R.S.I., Ibid.

74 Père Henri Gressien, né en 1858, jésuite en 1878, décédé en 1934 à Lyon. 1904-1907, Beyrouth : recteur de l'Université Saint-Joseph, chancelier de la Faculté orientale de Beyrouth. (Henry Jalabert, sj, op. cit.).

75 A.R.S.I., Carton 1007 : 1901-1918, Mission Syria, Farde Syria 1007-I, Différentes lettres du Père Cattin.

76 A.R.S.I., Ibid.

77 Carla Eddé, L'Université Saint-Joseph. Portrait d'une Université (Beyrouth : Presses de l'Université Saint-Joseph, Janvier 2000), 56.

78 Cahier des consultes de l'Université Saint-Joseph 1880-1920. 2 août 1911.

79 Ibid.

80 Ibid.

81 Père Claudius Chanteur, né en 1865 dans l'Isère, jésuite en 1888, décédé en 1949 au Caire. Préfet du Collège d'Alexandrie (1903-1905). Rentre en France. Provincial de Lyon (1912-1918), il se voit demander par l'Université d'Etat de Lyon la collaboration de l'Université Saint-Joseph pour la fondation à Beyrouth des Écoles de droit et d'ingénieurs et parvient, en dépit de l'anticléricalisme officiel, à faire respecter le rôle de la Compagnie de Jésus dans l'œuvre commune. Supérieur de la Mission (19181921, 1927-1933), Recteur de l'Université Saint-Joseph (1921-1927), Supérieur et Chancelier de la Faculté de Médecine de Beyrouth (1927-1942). (Henry Jalabert, sj. op. cit.).

82 Pour plus de détails sur cette problématique voir : A.J.V.. Coll. PRAT N.C. Tome 28-2.

83 A.J.V., Coll. PRAT N.C. Tome 28-2. P. 211.

84 A.J.V., Ibid. 
85 La leçon inaugurale du Professeur Huvelin est reproduite en tête des Mélanges à la mémoire de Paul Huvelin. Livre du 25ème anniversaire de l'Ecole Française de Droit de Beyrouth. Paris : Sirey, 1938.

86 Voir une partie du discours inaugural prononcé par Paul Huvelin dans Munir Antonios Bashshur. Op.Cit. P.48.

87 Pour plus de renseignements sur l'histoire du Droit libanais voir : Antoine Elias El-Gemayel (Edited). The Lebanese Legal System. Volume 1. Washington D.C. : International Law Institute in Cooperation with Georgetown University, 1985.

88 Les Jésuites en Syrie, 1831-1931.op.cit., Fascicule 3 : L'Ecole de Droit, 7.

89 A.J.V., Carton RPO 51 : Université Saint-Joseph, Faculté de droit.

90 A.J.V., Ibid.

91 A.J.V., Carton RPO 61 : Université Saint-Joseph, École d'ingénieurs.

92 A.J.V., Coll. PRAT N.C. Tome 28-2, 211.

93 A.J.V., Carton RPO 61 : Université Saint-Joseph, École d'ingénieurs.

94 A.J.V., Coll. PRAT N.C. Tome 28-2, 179.

95 Voir à ce sujet : ACG Beyrouth, Carton: A137, 1885/86, Dossier: «Traitements des Professeurs ». Ibid., Carton: A235, 1913, Carton: A238, 1913, Carton: A293, 1903/04, Carton: A303, 1908/1909, Carton: A337, 1882/1914.

96 A.J.V., Coll. PRAT N.C. Tome 28-2, 427.

97 Les Jésuites dirigeaient à l'époque dans leurs diverses missions, plus de vingt observatoires certains jouissant d'une célébrité universelle : Manille, Cuba, Zi-Ka-Wei, Tananarive....

98 Père Bonaventure Berloty, né en 1856 à Lyon, jésuite en 1874, décédé en 1934 à Beyrouth. Mathématicien. Fondateur de l'observatoire de Ksara. Docteur ès sciences mathématiques en 1866. Professeur aux Facultés catholiques de Lyon (1891-1901). En Orient depuis 1902. Fondateur en 1907 de l'observatoire de Ksara dont il avait choisi le site et acquis l'équipement et qu'il réorganisa de sa ruine totale après la guerre de 19141918. Directeur de l'observatoire jusqu'en 1925, responsable ensuite de la sismologie. (Henry Jalabert, sj, op. cit.).

99 Les Jésuites en Syrie, 1831-1931, op. cit., Fascicule 8 : L’Observatoire de Ksara et les services de météorologie, 7-8.

100 Ibid.

101 A.J.V., Collection PRAT, Tome 28, 2 : Missions de Syrie et d'Arménie 1906-1920.

102 R.P. Delore. "La bonne presse au Liban ", Les Missions catholiques, Tome 45 de 1913, 201-213.

103 "L'imprimerie catholique de Beyrouth ", Les Missions catholiques, Tome 14 de 1882, 616-618.

104 Père Edouard Billotet, né en 1812 en Haute-Saône, décédé en 1860 à Zahle. Supérieur de la Mission (1850-1859). Il s'embarque pour Beyrouth fin 1845 et adopte désormais le nom d'Abouna Hanna (Père Jean). Professeur à l'École de Beyrouth, il y introduit l'usage de la première communion solennelle. Il est à l'origine de l'imprimerie catholique et développe également le Séminaire-Collège de Ghazìr, dont il fait nettement séparer les deux sections. Il périt dans les massacres de 1860 à Zahle "parce que chrétien ". (Henry Jalabert, sj, op. cit.) ; Pour plus de détails voir la liste d'une dizaine de livres ou de brochures en arabe dans: Les Jésuites en Syrie, 1831-193, op. cit., Fascicule 6: Les Euvres de presse, 10.

105 Jean-Baptiste Piolet, "L'Université Saint-Joseph à Beyrouth, à l'occasion de son cinquantenaire ", Revue d'Histoire des Missions Tome III du $1^{\mathrm{er}}$ mars 1926. (Paris : Editions SPES), 84.

106 Frère Antoine Tallon, né en 1816 à Lyon, jésuite en 1838, décédé en 1888 à Beyrouth. Arrive à Beyrouth en 1853, il semble avoir été chargé dès son arrivée de la petite imprimerie fondée en 1852, mais n'est désigné comme “imprimeur" au catalogue qu’à partir de 1861, jusqu'en 1887. (Henry Jalabert, sj, op. cit.) 
107 Père Billotet. [Sans Titre]. Bulletin de l'Euvre des Ecoles d'Orient. Numéro d'avril 1858.

108 Père Joseph Heury, né en 1824 en Avignon, jésuite en 1842, décédé en 1897 à Zahle. Il fut envoyé enseigner la philosophie et les mathématiques à Ghazīr (1851-1852); après quoi il reprit l'étude de la théologie morale, seul à Beyrouth (1852-1853), puis avec les séminaristes à Ghazīr (1853-1854), en même temps qu'il étudiait l'arabe. Professeur à Ghazīr, missionnaire à Saida, procureur à Beyrouth, chargé une année à l'imprimerie catholique (1860-1861). Ses dernières années se passent à Zahle, où il est supérieur (1890-1893), puis père spirituel. Sa notice révèle qu'on lui doit le premier dictionnaire arabe-français qui a été imprimé en Orient. (Henry Jalabert, sj, op. cit.).

109 Père Philippe Cuche, né en 1818 dans le Doubs, jésuite en 1843, décédé en 1895 à Bikfaya. Vice-recteur de Ghazir (1858-1861), fondateur des Résidences d'Alep (1873) et de Homs (1882). Bon écrivain arabe. Directeur du Bachir en 1877 et en 1881, auteur de "plusieurs articles à sensation ». Auteur aussi d'un dictionnaire arabe-français. Supérieur de la Résidence de Bikfaya depuis 1892 où il y mourut. (Henry Jalabert, sj, Ibid.).

110 [Sans Titre]. Bulletin de l'Euvre des Ecoles d'Orient. Numéro de mai 1863. P.288.

111 Frère Marie Elias, né en 1840 à Caifa, jésuite en 1862, décédé en 1901 à Tanail. Le Frère Marie Elias est envoyé en France pour des raisons de sécurité, il entre dans la Compagnie en 1862 et revient alors en Orient. Forgeron, puis chargé en 1869 de l'imprimerie catholique, il suit des stages à Paris et à Londres et révolutionne à son retour l'impression arabe en fondant ensemble la consonne et chacun des accentvoyelles, innovation adoptée depuis par toutes les imprimeries du monde arabe. Grâce à sa formation à Paris, il publia une version arabe de la Bible (1875), pour l'opposer à la version protestante. Il dirigea les ateliers de l'imprimerie jusqu'à sa mort, pendant trente ans, et en fit la maison la mieux équipée de l'Empire ottoman. En 1899, l'imprimerie fit l'acquisition d'une troisième machine motrice de la force de douze chevaux. C'est la dernière grande installation qu'il fut donné au Frère Marie Elias avant sa mort en 1901 à Tanail. (Henry Jalabert, sj, op. cit.).

112 Henri Charles, sj, Jésuites missionnaires. Syrie. Proche-Orient (Paris : Editeur Beauchesne, 1929), 31.

113 Les Jésuites en Syrie, 1831-1931, op. cit., Fascicule 6 : Les Euvres de presse, 37.

114 Belin (Consul général de France à Constantinople). "L'imprimerie des Jésuites à Beyrouth ", Les Missions catholiques. Tome 7 de 1875, 27-28.

115 Les Jésuites en Syrie, 1831-1931, op. cit., 12.

116 "L'Imprimerie catholique de Beyrouth et son œuvre en Orient (1853-1903)». Fascicule supplémentaire des relations d'Orient (Bruxelles : Imprimerie Polleunis, Ceuterick et Lefebvre, 1903), 143 p.

117 Père Jean-Baptiste Belot, né en 1822 à Lux, jésuite en 1842, décédé en 1904 à Beyrouth. En Orient à partir de 1865. Directeur de l'imprimerie Catholique (18681898; pour l'arabe seulement, 1898-1904) : assisté du Frère Marie Elias qui en dirige les ateliers, il lui donne le grand essor. Auteur d'une vingtaine d'ouvrages en arabe, originaux ou traductions, de spiritualité, vie chrétienne, Écriture Sainte (une Bible des enfants en trois volumes) et de deux dictionnaires : arabe-français, al-Faraid et françaisarabe, le Belot. Fondateur en 1870 du Bachir, premier journal catholique en langue arabe. Il décéda en 1904 à Beyrouth. (Henry Jalabert, sj, op. cit.).

118 Père Donat Vernier, né en 1838 dans le Doubs, jésuite en 1855, décédé en 1917 à Beyrouth. Scolastique à Ghazīr (1859-1868), en France pour la théologie. Revenu en Orient en 1873. Travailleur acharné, auteur d'une grammaire arabe en français, d'ouvrages en arabe (au style assez archaïque), d'une Histoire du Patriarcat arménien catholique. (Henry Jalabert, sj, Ibid.).

119 Père Joseph-Gabriel Hava, né en 1851 à Marseille, jésuite en 1882, décédé en 1916 au Liban. Grec catholique, il connaissait de nombreuses langues. Envoyé à Beyrouth en 
1883, il dirige avec un autre père la congrégation des ouvriers de Beyrouth (1889-1891) et est dès lors missionnaire à partir des diverses maisons du Liban. "Ecrivain " (Scriptor) à Beyrouth en 1895-1896, l'année sans doute où il mit au point son dictionnaire arabeanglais, très apprécié, paru en 1899. (Henry Jalabert, sj, Ibid.).

120 "L'imprimerie catholique de Beyrouth et son œuvre en Orient (1853-1903)". op. cit.

121 Ibid.;

Pour plus d'informations sur les publications périodiques de l'imprimerie catholique de Beyrouth voir : A.S.V., Carton numéro 199: 1900-1913 : Relazioni e Corrispondenza della Delegazione Apostolica con i Padri Gesuiti, Lettre du Père Cattin datant du 28/9/1903;

A.P.F., Carton numéro 2 : 1874-1880 : Scritture Riferite nei Congressi Siria, Lettre du $22 / 1 / 1880$.

122 "La presse protestante et le Bachir ", Les Missions catholiques. Tome 4 de 1872. 275276, 517-518.

123 Voir la biographie du Père Ambroise Monnot au début de cet article.

124 A.R.S.I., Carton 1005 : 1869-1890, Mission Syria, Farde Syria 1005-II, Document numéro 45 datant de 1882 .

125 Voir un exemple de Al-Bachir conservé à Rome : A.R.S.I., Carton 1005 : 1869-1890, Mission Syria, Farde Syria 1005-VI, Document numéro 29 datant du 13/1/1885.

126 Jean-Baptiste Piolet. op. cit., 87.

127 En 1903 par exemple, par suite des exigences de la censure de Constantinople, l'almanach a dû supprimer quelques-uns des renseignements qu'il avait coutume de donner à ses lecteurs, comme la liste des autorités civiles et militaires du pays. Voir aussi : A.R.S.I., Carton 1007 : 1901-1918, Mission Syria, Farde Syria 1007-I, Lettre numéro 5 du Père Cattin datant du 5/5/1902.

128 Al-Machriq a eu un précurseur, la revue parut sous le titre de L'Eglise catholique le $1^{\text {er }}$ janvier 1888.

129 Voir aussi sur la revue Al-Machriq : A.S.V., Carton numéro 199: 1900-1913 : Relazioni e Corrispondenza della Delegazione Apostolico con i Padri Gesuiti, Lettre du Père Cattin datant du 28/9/1903.

130 "L'imprimerie catholique de Beyrouth et son œuvre en Orient (1853-1903)», op. cit., 117.

131 Pour plus de détails sur les articles de la revue Al-Machriq voir : "Limprimerie catholique de Beyrouth et son œuvre en Orient (1853-1903) ", Ibid., 118-133.

132 Cette revue était alors le trait d'union entre l'Orient et l'Europe savante et avait deux catégories d'abonnés : les orientalistes et les lecteurs orientaux.

133 En effet, dans les pays européens, ce sont surtout les centres intellectuels d'Allemagne, d'Autriche et d'Italie qui fournissent le plus grand nombre de lecteurs. Voir aussi : "Un Centro missionnario di Studi superiori in Oriente, l'Université Saint-Joseph di Beirut". Rivista dell' Unione missionaria del Clero. Gennaio 1927.

134 Lettre adressée par le professeur M. Charles Nallino au Père Louis Cheikho cité dans : "L'imprimerie catholique de Beyrouth et son œuvre en Orient (1853-1903) », op. cit., 133.

135 Jean-Baptiste Piolet, op. cit., 91.

136 A.R.S.I., Carton 1007 : 1901-1918, Mission Syria, Farde Syria 1007-III, Lettre numéro 51 de 1914.

137 Nous connaissons tous les évènements de la guerre grâce au diaire du R.P. Paul Mattern, qui, en tant qu'Alsacien a le droit de rester sur place. Voir à ce sujet dans les Archives Jésuites de Vanves : A.J.V., Carton RPO 1 : Collection Jallabert, 1963 (Archives de la Curie de Rome), diaire personnel du R.P. Paul Mattern, 1914-1918.

138 A.J.V., Carton RPO 43 : Problèmes Communs, Chemise 2. 
139 "Il nous est possible de calculer les milliers d'élèves qui ont passé par le Collège et y ont reçu une formation ". Pour plus de renseignements sur l'état de la Mission après la Première Guerre mondiale voir : A.J.V., Collection Jalabert. Ce fond sur la Mission de Syrie se rapporte surtout au $\mathrm{XX}^{e}$ siècle et traite aussi de questions politiques et administratives.

140 Révérend Père Maître. "Lettre sur le Séminaire oriental ", Les Missions catholiques, Tome 36 (1904), 241-242.

141 "Les Jésuites en Syrie : Le bilan d'un centenaire. Le problème missionnaire tel qu'il se pose en Syrie ", Lettres de Fourvière (Province de Lyon) (Lyon : Imprimerie L. Bascou. Troisième série, 4 (novembre 1931), 255.

142 "Entre 1887 et 1912, 354 médecins et 87 pharmaciens sont sortis de la F.F.M.». (A.J.V., Carton RPO 43 : Problèmes communs, Chemise 2.).

143 «Les Jésuites en Syrie : Le bilan d'un centenaire. Le problème missionnaire tel qu'il se pose en Syrie ", op. cit., 255.

144 "Depuis sa création jusqu'aux années 1930, l'École de droit comptait 210 diplômés et l'École d'Ingénieurs 61 apprentis-ingénieurs ". (A.J.V., Carton RPO 51 : Université Saint-Joseph, Faculté de droit, Ibid., Carton RPO 61 : Université Saint-Joseph, École d'ingénieurs).

145 Voir les chiffres trouvés dans les Archives Jésuites de Vanves, les Lettres de Fourvière et Les Missions catholiques.

146 "Feuilleton. L'Université Saint-Joseph à Beyrouth ». Les Missions catholiques (Bulletin hebdomadaire illustré de l'œuvre de la propagation de la foi). Lyon, Paris, Bruxelles. Tome huitième de janvier-décembre 1876, 506-507.

147 Voir à ce sujet les deux livres de Henri Lammens : Henri Lammens. La Syrie, précis historique. Deux volumes. Beyrouth, 1921 et Henri Lammens. La Syrie et son importance géographique. Louvain, 1904.

148 Asher Kaufman. "Phoenicianism : The Formation of an Identity in Lebanon in 1920". Middle Eastern Studies. Vol. 37 (1). January 2001, 173-194.

149 Asher Kaufman écrit sur ce sujet: "When Greater Lebanon was established in 1920, the first functionaries of the Lebanese State were chosen primarily among the Saint Joseph graduates. Thus, a strong link was established between the Jesuits of Saint Joseph and the new Lebanese State". (Asher Kaufman. Ibid., 177.). Sur les relations entre l'Université Saint-Joseph et l'État voir : David A. Kerr. The Temporal Authority of the Maronite Patriarchate. Thèse de doctorat, St. Antony's College. Oxford : 1973, 151; Iskandar alRiyashi. Ru'asa' Lubnan Kama 'Araftuhum. Bairūt : 1961, 15. 